\title{
REVIEW
}

\section{Imaging modalities in hand osteoarthritis - status and perspectives of conventional radiography, magnetic resonance imaging, and ultrasonography}

\author{
Ida K Haugen* and Pernille Bøyesen
}

\begin{abstract}
Hand osteoarthritis $(\mathrm{OA})$ is very frequent in middle-aged and older women and men in the general population. Currently, owing to high feasibility and low costs, conventional radiography (CR) is the method of choice for evaluation of hand OA. CR provides a two-dimensional picture of bony changes, such as osteophytes, erosions, cysts, and sclerosis, and joint space narrowing as an indirect measure of cartilage loss. There are several standardized scoring methods for evaluation of radiographic hand OA. The scales have shown similar reliability, validity, and sensitivity to change, and no conclusion about the preferred instrument has been drawn. Patients with hand OA may experience pain, stiffness, and physical disability, but the associations between radiographic findings and clinical symptoms are weak to moderate and vary across studies. OA is, indeed, recognized to involve the whole joint, and modern imaging techniques such as ultrasound (US) and magnetic resonance imaging (MRI) could be valuable tools for better evaluation of hand OA. Standardized scoring methods have been proposed for both modalities. Several studies have examined the validity of US features in hand OA, whereas knowledge of the validity of MRI is more limited. However, both synovitis (detected by either US or MRI) and MRI-defined bone marrow lesions have been associated with pain, indicating that treatment of inflammation is important for pain management in hand OA. Both US and MRI have shown better sensitivity than $\mathrm{CR}$ in detection of erosions, and this may indicate that erosive hand OA may be more common than previously thought.
\end{abstract}

\section{Introduction}

Osteoarthritis (OA) is the most prevalent musculoskeletal disease in developed countries, and the hands are frequently involved [1]. Despite the high prevalence, hand $\mathrm{OA}$ is receiving less attention compared with OA of the weight-bearing joints such as the knees and hips. Typically, the distal interphalangeal (DIP) joints and the thumb base and, to a lesser extent, the proximal interphalangeal (PIP) joints are affected [1]. Patients with hand OA can experience considerable pain, stiffness, and disability with a high impact on health-related quality of life, but there is currently no structure-modifying treatment. Development of new treatments requires knowledge of the natural disease course and use of reliable and sensitive outcome measures [2].

*Correspondence: haugen_ida@hotmail.com

Department of Rheumatology, Diakonhjemmet Hospital, P.O. Box 23, Vinderen, 0319 Oslo, Norway
Outcome measures in OA usually include evaluation of pain and disability and imaging of joint structural changes. Currently, conventional radiography (CR) is the most economical, feasible, and easily available imaging modality for assessment of structural hand OA features. However, since OA is increasingly recognized to involve the whole joint, modern imaging techniques such as ultrasonography (US) and magnetic resonance imaging (MRI) have been introduced recently for evaluation of hand OA.

\section{Conventional radiography}

Owing to high availability, good feasibility, and low costs, $\mathrm{CR}$ is still the gold standard for morphological assessment of hand OA [2]. The prevalence estimates of radiographic hand OA vary across studies, and this may be due to differences in risk factors between populations or possibly different definitions of disease. Currently, there is no established gold standard for the definition of radiographic hand $\mathrm{OA}$, and the studies also differ in 
regard to grading systems that are used and the definitions of radiographic hand $\mathrm{OA}[3]$.

\section{Common features of hand osteoarthritis}

CR provides a two-dimensional picture of bony changes, such as osteophytes, erosions, cysts, and sclerosis, and joint space narrowing (JSN) as an indirect measure of cartilage loss (Figure 1).

Osteophytes can be divided into 'true' intra-articular osteophytes and traction spurs. The 'true' intra-articular osteophytes are located at the joint margins [4] and can easily be seen on CR with a traditional posteroanterior view. The traction spurs, on the other hand, are located at the insertion of the extensor tendon or along the midshaft and are most easily seen on CR with an oblique or lateral view. Whether these enthesophytic changes are related to $\mathrm{OA}$ is not entirely clear, and previous studies have suggested that these changes are related mainly to age and local biomechanical factors and not a systemic enthesopathy $[5,6]$.

Radiographic measurement of JSN is currently recommended as the imaging endpoint for clinical trials of disease-modifying OA drugs [7]. The cartilage cannot be directly assessed by CR and therefore is indirectly judged by the inter-bone distance. The evaluation may be affected by positioning of the hands (for example, flexion deformities) and is further complicated by erosive development in the finger joints, which may lead to increased joint space width (JSW) (that is, pseudo-widening) despite worsening of disease.

Radiographic erosions in hand $\mathrm{OA}$ are seen as bone damage in the central part of the joints with a typical seagull wing configuration. They typically occur in the DIP and PIP joints [1] but have also been described in the thumb base joints [8]. Longitudinal studies have shown that JSN precedes the erosive development, suggesting that local biomechanical factors are important for the erosive development $[9,10]$. These findings may suggest that erosive hand OA represents severe hand OA rather than a separate disease entity.

Cysts are seen as loss of the trabecular structure, whereas sclerosis is seen as increased density on CR. Both features may be related to bone remodeling. Histological studies have shown that areas with sclerosis are characterized by increased thickness of the subchondral plate and the trabeculae, and this may indicate repair of bone trauma [11].

\section{Scoring systems}

Here, we will briefly present the most widely used scoring systems for assessment of radiographic hand OA. Currently, there is no consensus on the preferred scale. The first proposed radiographic scoring system was the Kellgren and Lawrence (K\&L) scale [12], which is still the most widely used [3]. The K\&L scale is a global scale that grades OA on a range from 0 to 4 points (a grade of at least 2 represents definite $\mathrm{OA}$ ) on the basis of the presence/severity of osteophytes/ossicles, JSN, sclerosis, pseudocystic areas, and altered shape of bone ends. Different descriptions of the grades for various joint groups and across publications have caused confusion in how to interpret the different grades [13,14]. Furthermore, the K\&L scale has been criticized for too much emphasis on osteophytes [15] as narrowed/sclerotic joints cannot be classified as having OA unless osteophytes are present. Thus, several studies have used modified K\&L scales to overcome these deficiencies.

Assessment of individual features instead of a global score may also optimize agreement, and the Osteoarthritis Research Society International (OARSI) atlas is most frequently used [16,17]. With this atlas as a reference, the presence and severity of individual features are assessed on semi-quantitative scales. However, scoring of individual features may be more time-consuming, and we have no agreed-upon definition of hand OA on the basis of individual radiographic features.

Verbruggen and colleagues [9] suggested an alternative approach and developed a numerical scoring system for the anatomic evolution of erosive and non-erosive hand OA. This system is based on an underlying assumption of hand $\mathrm{OA}$ as a disease that undergoes predictable phases. The scale is most suitable for the evaluation of erosive hand OA as the progression of JSN and osteophytes does not necessarily mean progression in terms of the proposed phases.

Reliability exercises have shown that hand OA can be assessed reliably by CR. However, despite the limitations of the K\&L scale, studies have not been able to show better reliability with other scoring systems $[18,19]$. Hence, based on the different character of the scales, the choice of scale rather depends on the study objective.

Radiographic hand OA is usually a slowly progressive disease $[1,20,21]$. CR is most likely not a sensitive measure of hand OA progression or, as previously shown, of knee OA progression [22]. Newly proposed computerized methods for quantification of JSW may provide a more sensitive set of tools for the assessment of OA progression over time [23-25], but longitudinal studies are needed.

\section{Associations with pain and physical function}

Studies have suggested a positive association between radiographic hand OA and hand pain, but the strength of the association varies across studies [26]. The associations between radiographic hand $\mathrm{OA}$ and hand disability are more inconsequential, ranging from no to moderate associations [26]. Patients with erosive hand OA experience more pain and physical disabilities compared with patients with non-erosive hand OA, but the difference 


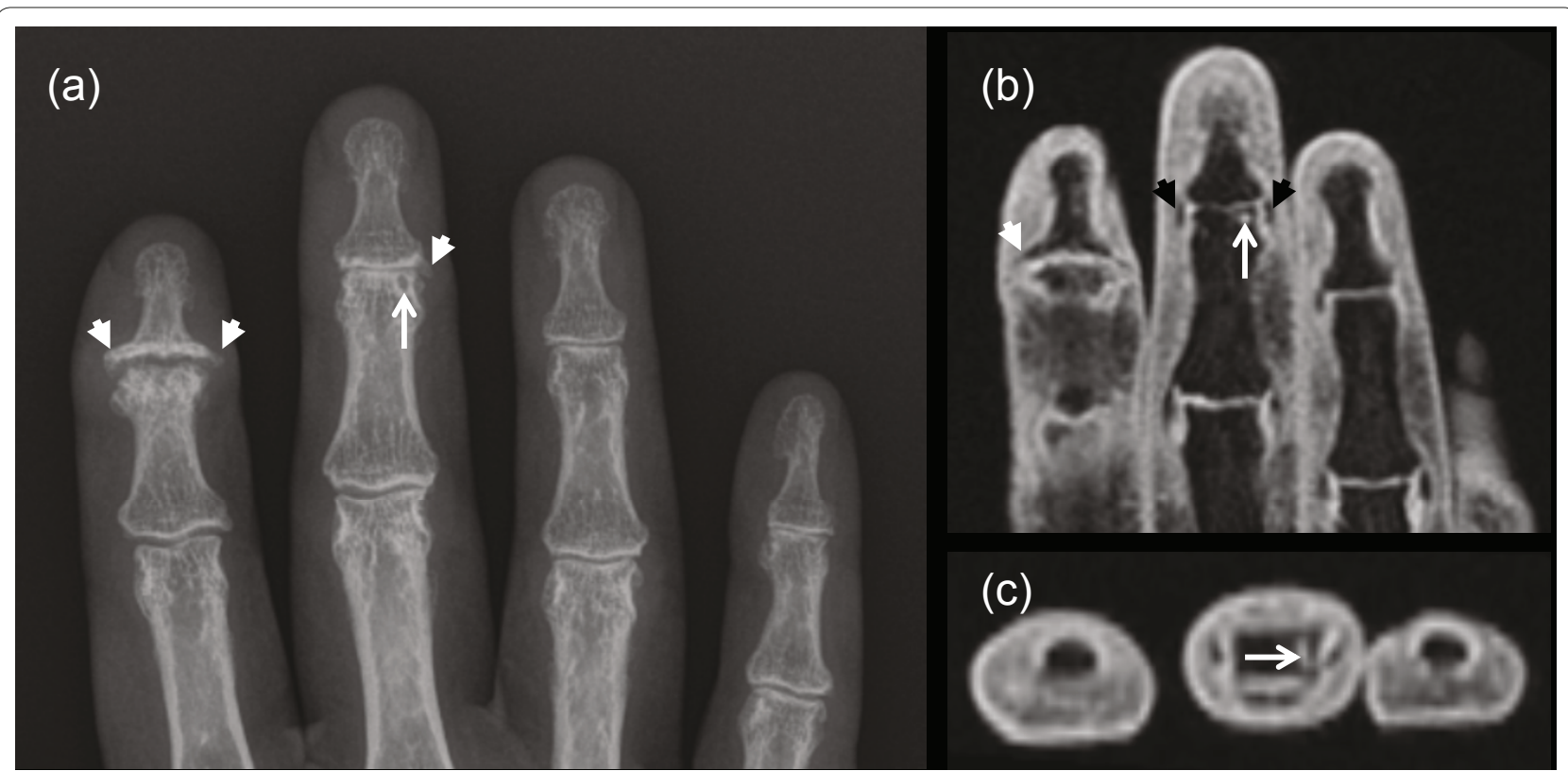

Figure 1. Conventional radiography (CR) and magnetic resonance imaging (MRI) (coronal/axial T1-weighted fat-suppressed images) of the right hand. Both CR (a) and MRI $(\mathbf{b}, \mathbf{c})$ show severe osteoarthritis with osteophytes (white arrowheads) and central collapse of the joint plate in the 2nd distal interphalangeal (DIP) joint. Both MRI and CR show severe joint space narrowing in the 3rd DIP joint. The osteophytes are more easily seen on CR, whereas MRI shows the collateral ligaments (black arrowheads). CR shows a cyst-like lesion (white arrow), which on MRI seems to be an erosion (that is, a cortical break in the axial plane).

seems to be due at least partly to a higher burden of disease in the erosive patients. Kortekaas and colleagues [27] just recently showed that hand osteophytes and JSN were associated with tenderness in the same joint independently of each other and synovitis. However, cartilage is aneural and cannot be a direct source of pain, and this means that the association is possibly mediated by bone damage (as a consequence of decreased bone unloading associated with cartilage loss).

Despite positive associations in cross-sectional studies, longitudinal studies have not been able to show any association between radiographic progression and clinical deterioration [19-21]. In general, studies focusing on the amount of structural features or the number of affected joints and the relation to hand pain and functioning are less likely show associations with measures of pain and functioning compared with analyses performed at the individual joint level. First of all, psychosocial factors affect the self-report of symptoms [28]. Furthermore, radiographic studies are limited by the fact that pain in hand OA is related to not only structural abnormalities but also pain perception and inflammation.

\section{Future perspectives}

Standardization of the definition of radiographic hand OA with respect to scoring methods, joints under evaluation, and the required number of affected joints could possibly reduce the variations across studies. Although the K\&L scale has the benefit of being simple and is well known in the research community, the system has several limitations. However, comparative studies have not been able to conclude about the preferred instrument. Quantitative measurement of JSW may be a more sensitive measure of progression in hand OA, but the sensitivity to change must be explored in future longitudinal studies.

\section{Ultrasonography}

In recent years, US has gained acceptance as a useful tool for the assessment of inflammation in the finger joints of patients with rheumatoid arthritis. Lately, the prevalence, validity, and reliability of US features have also been studied in patients with hand OA. US has the advantage of providing a multiplanar dynamic image and does not involve any radiation and can be performed in the examination room without any inconvenience for the patient. Optimal visualization is achieved by both longitudinal and transverse scanning of the dorsal aspects with the joint in full flexion and of the volar aspects with the joints in neutral position [29].

\section{Common features of hand osteoarthritis}

US allows visualization of a wide spectrum of hand OA features, including osteophytes, marginal erosions, and synovitis (Figure 2). US may, therefore, be a feasible tool for visualization of inflammation in patients with hand OA. One of the disadvantages of US is the inability of its 


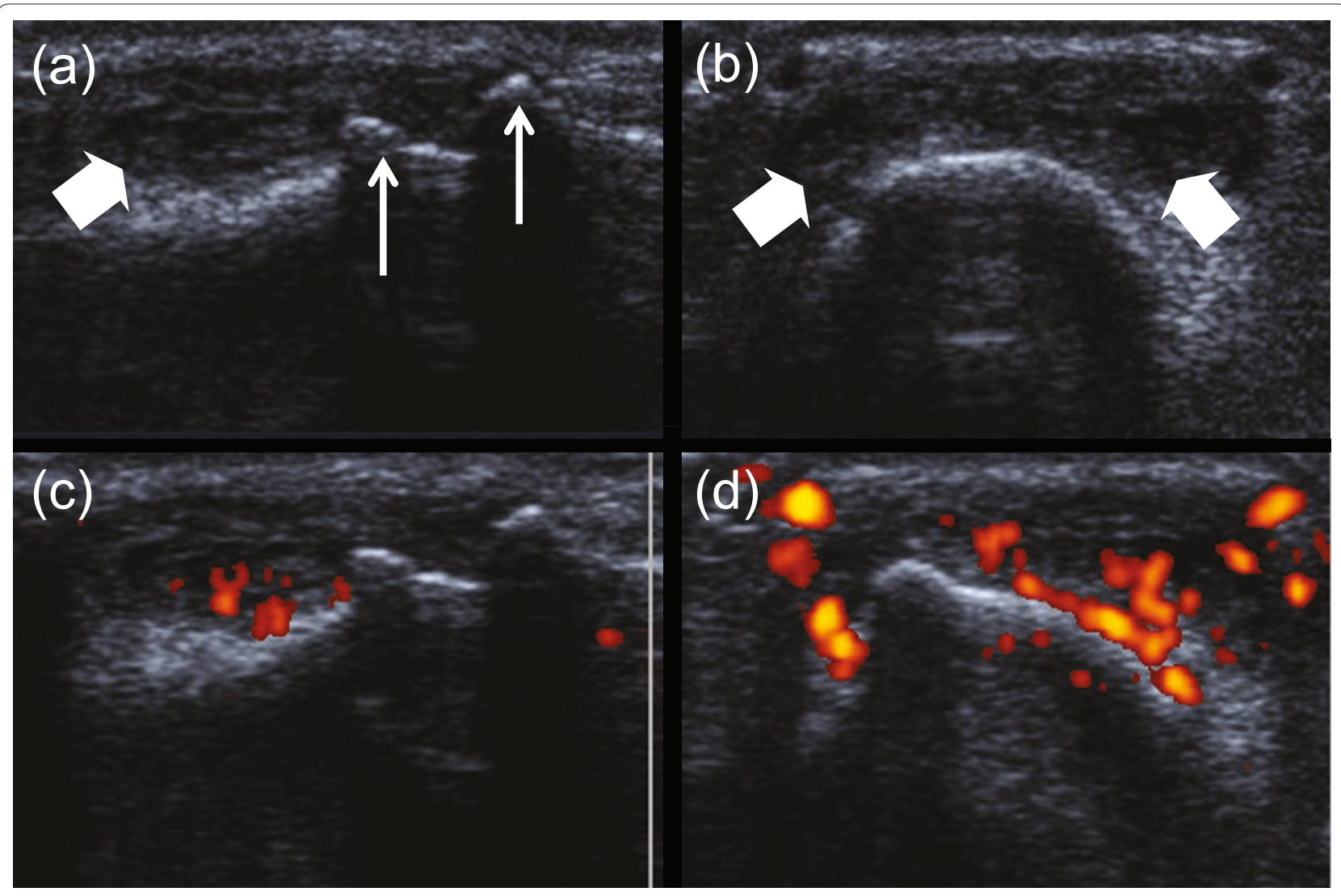

Figure 2. Ultrasonography of the $\mathbf{2}$ nd proximal interphalageal joint. The joint is visualized in sagittal $(\mathbf{a}, \mathbf{c})$ and axial $\mathbf{( b , d )}$ scans. In a gray-scale image (a), proximal and distal osteophytes are visible (arrows). Gray-scale synovitis is visible in both sagittal (a) and axial (b) planes (arrowheads). Power Doppler signal is visible in (c) and (d).

beam to penetrate bony cortex. Thus, owing to the joint anatomy, visualization of the articular cartilage and bone damage is restricted mostly to the peripheral parts [29]. Overlying osteophytes, which disturb the acoustic window, further complicate the evaluation. In severely damaged joints, it might be difficult to determine the point at which an erosion starts and an osteophyte ends.

Most US studies of patients with hand OA have reported a high prevalence of gray-scale synovitis [30-33], whereas power Doppler activity has been less frequent $[30,32,33]$. Kortekaas and colleagues [33] found that both gray-scale synovitis and power Doppler activity were present in the majority of patients with hand OA, but the number of joints with power Doppler activity was considerably lower than the number of joints with grayscale synovitis. However, other studies have demonstrated greater similarity in the frequencies of power Doppler activity and gray-scale synovitis $[31,34]$. These variations across studies may be due to differences in study populations or US techniques.

Erosive OA is often called 'inflammatory' OA. Mancarella and colleagues [34] found a higher amount of power Doppler activity, synovial hypertrophy, and joint effusion in patients with radiographic erosive OA joints in comparison with patients with radiographic non-erosive OA joints. Synovitis seems to be most prevalent in joints with active erosions, whereas the prevalence is lower in joints that are remodeled [35]. These findings may indicate a burnout of inflammation in late stages, but this hypothesis needs to be confirmed in longitudinal studies.

\section{Scoring system}

One preliminary US scoring system has been developed for hand OA. A group of experts in the fields of OA, US, and outcome measures agreed upon a scoring system for hand OA features, including assessment of synovitis (gray-scale hypertrophy/effusion and power Doppler) and osteophytes on semi-quantitative scales [36]. Erosions, cartilage assessment, and JSN were not included in the scoring system, because of concerns about reliable definitions, acquisition, currently available US technology, and feasibility related to duration of scanning.

Dependency on the US operator represents one of the major limitations of US examination. Several studies report inter-reader reliability based on stored images, and this limits the variability related to the performance 
and technique of the US examination. A large reliability exercise was arranged in order to test the reliability of the preliminary US scoring system [36]. Despite divergent results, the authors concluded that the results were satisfactory and that the proposed scoring system could provide a good basis for further development of a US outcome tool.

\section{Validity against other imaging modalities}

The validity of US against other imaging modalities (especially CR) has now been tested in several studies. The first report comparing US and CR found that CR was more sensitive than US in detection of erosions and indicated that the interposition of osteophytes could limit the acoustic window of US [37]. However, later reports have shown that US is most sensitive in the detection of erosions [31,38] as well as osteophytes and JSN $[31,38,39]$. The higher sensitivity is probably due to the multiplanar visualization of the joint by US. Erosions were found not only in the central part of the joints but also in the peripheral sections [31]. Furthermore, some radiographic cysts appeared to be erosions as demonstrated by US [31]. However, it must be noted that erosions may be difficult to assess in joints with severe OA with excessive bone formation and deformities, which limit the acoustic window.

Estimation of JSN by US may be problematic as only the peripheral inter-bone distance can be documented and overlying osteophytes may further decrease the acoustic window [39]. However, in two studies, the investigators measured the cartilage thickness quantitatively and found significant association between lower US-defined cartilage thickness and radiographic severity, JSN (semi-quantitative scale), and JSW (quantitative scale) [34,40].

So far, few studies have compared the findings by US and MRI, but the current results support the use of US as a valid instrument. Wittoek and colleagues [38] compared US against MRI as reference and found good agreement for both structural features and inflammation. Iagnocco and colleagues [41] used US and MRI fusion imaging and found that that hyperechoic prominences seen by US corresponded to osteophytes seen by MRI. However, the optimum application of this technique has not yet been determined, and the extra cost and time limit its use.

\section{Associations with pain and physical function}

Several studies have reported that US pathological features such as gray-scale synovitis, power Doppler signal, and osteophytes are significantly associated with pain at the individual joint level $[27,30,33]$. Kortekaas and colleagues [33] showed that gray-scale synovitis was significantly associated with pain. However, the analyses were not adjusted for structural features.
As discussed in the section on radiographic features and measures of pain and physical function (see previous 'Associations with pain and physical function' section), studies are less likely to show significant associations when the analyses are performed at the patient level instead of the individual joint level. Analyses of the association between US features (number of affected joints or sum scores) and measures of global hand pain, stiffness, and physical disability have revealed conflicting results. In line with analyses at the individual joint level, Kortekaas and colleagues [33] found significant associations between gray-scale synovitis and pain, stiffness, and physical disability. However, other studies have found no significant associations [30,42]. Koutroumpas and colleagues [42] found that clinically inflamed joints, but not US inflammation, were significantly correlated to physical limitations. These findings may indicate that US detects low-grade inflammation, which possibly does not reflect OA pathology.

The association between US features and pain should also be explored in longitudinal studies, but we are aware of only one observational study of patients with hand OA treated with intra-muscular metylprednisolone: Keen and colleagues [32] found a significant reduction in symptoms but no statistically significant reduction in US-detected synovial inflammation after 4 and 12 weeks. Interestingly, there was no association between reduction in symptoms and reduction in US inflammation. However, as this was an open-label study, one cannot rule out the possibility that the observed clinical response represented a placebo effect.

\section{Future perspectives}

The reliability of US scoring could be greatly enhanced by the development of a scoring atlas. To the best of our knowledge, there are no hand OA randomized controlled trials that use US as an outcome measure. Longitudinal studies are also required in order to study the association between US features and pain. Ultimately, the role of US scans in daily clinical practice needs to be addressed.

\section{Magnetic resonance imaging}

MRI is an established outcome measure in inflammatory diseases such as rheumatoid arthritis and knee OA and has increased our knowledge of disease mechanisms. With the use of MRI, OA is now recognized as a disease affecting the whole joint. Currently, only limited research on the prevalence, reliability, and validity of MRI-defined pathology in hand OA is available.

\section{Common features of hand osteoarthritis}

MRI has the ability of providing a multiplanar image of all joint components, including structural features such as osteophytes, cartilage, erosions/cysts, misalignment, 
and collateral ligaments and inflammatory features such as synovitis and tenosynovitis (Figure 1). MRI is the only modality that is able to show bone marrow lesions (BMLs), which have been proven as an important feature of structural progression and as a source of pain in knee OA [43].

Tan and colleagues [44] imaged DIP or PIP joints with OA by using high-resolution MRI and showed that virtually all structures were affected in both chronic and early OA, confirming that $\mathrm{OA}$ is a failure of the whole joint. BMLs, erosions, and synovitis were common features in this small study. The authors highlighted that collateral ligament abnormalities were universal in both chronic and early disease and demonstrated a close anatomic relation between the ligaments and erosions, BMLs, and bone formation. However, it should be noted that collateral ligament pathology was also frequent in the older controls, and whether these changes are only age-related or play a role in the pathogenesis of the disease is currently not clear. Furthermore, collagenous structures, such as the collateral ligaments, may demonstrate increased signal intensity because of the 'magic angle' phenomenon, which may lead to overestimation of ligament pathology [45].

The prevalence of MRI pathology in patients with hand OA has been investigated in several cohorts, of which the Oslo hand OA cohort is the largest [46]. In that study, the authors found a high prevalence of synovitis on the basis of enhancement of gadolinium [46]. Synovitis was also highly prevalent in joints without radiographic OA, and this is in line with previous observations in knee OA [47]. The high prevalence of synovitis has been confirmed in smaller cohorts $[38,48]$. However, minimal gadolinium enhancement may occur also in persons without $\mathrm{OA}$, and therefore we propose that synovitis not be scored as present unless there is an accompanying thickness of the synovium.

Haugen and colleagues [46] found a low prevalence of BMLs, in contrast to the high prevalence shown in the smaller studies $[38,48]$, and this could be due to lower field strength and poorer resolution. In the small finger joints, it is also important to be aware of partial volume artifacts that may mimic BMLs [49].

Patients with radiographic hand $\mathrm{OA}$, in comparison with those with non-erosive hand OA, usually have a higher burden of disease. Wittoek and colleagues [38] confirmed that MRI-defined erosions, synovitis, and BMLs were more frequent in patients with radiographic erosive disease than in patients with radiographic nonerosive disease.

\section{Scoring system}

Haugen and colleagues [50] recently proposed a preliminary extensive MRI scoring system with an accompanying atlas for hand OA. The system includes assessment of osteophytes, JSN, erosions, cysts, misalignment, synovitis, flexor tenosynovitis, BMLs, and collateral ligament pathology such as absence/discontinuity and BMLs at insertion sites. The score was developed for the DIP and PIP joints, and future studies must confirm whether the score can be similarly applied to the metacarpophalangeal (MCP) and thumb base joints.

Haugen and colleagues [50] showed good intra- and inter-reader reliability for the assessment of most features in the proposed MRI scoring system. Good reliability of the scoring system has been confirmed in another cohort [48]. These positive results suggest that MRI can be a reliable tool for assessment of OA pathology in the small finger joints.

\section{Validity against histology and other imaging modalities}

The validity of MRI features in hand OA has been tested against histology and other imaging modalities [38,46, 48,51-53]. Lewis and colleagues [51] compared MRI features and corresponding histological findings in three fingers from cadavers. Osteophytes and cartilage loss could be seen on histological sections, whereas only the largest structures were visualized by MRI. Tan and colleagues [52] recently combined high-resolution MRI and cadaveric histological studies in order to better understand the role of the collateral ligaments in the pathogenesis of OA. However, this study was limited by the fact that the MRI scans and histological sections were not from the same patients. The histological sections showed OA degenerative changes, fissuring, and cell clustering in the collateral ligaments and the enthesal organ, and the authors suggested that the MRI-defined ligament abnormalities were caused by degenerative changes.

Grainger and colleagues [53] were the first to report that high-resolution MRI was more sensitive than CR in detection of erosions and especially in marginal erosions in hand OA. These findings have since been confirmed by several studies using conventional MRI [38,46,48]. MRI was able to visualize more joints with erosions in patients with radiographic erosive hand OA but was also able to detect joints with erosions in patients with radiographic non-erosive disease. However, at this time, we do not know the prognostic value of these marginal erosions, and longitudinal studies are needed.

Two studies have shown that MRI is more sensitive than CR in detection of osteophytes [46,48], and this may be due to the multiplanar demonstration of the joint by MRI. CR poorly visualizes bone formation located at the insertion of the extensor tendon unless there are oblique or lateral views. However, demonstration of osteophytes requires good contrast against adjacent structures because of the signal void of the cortical bone [38]. 


\section{Associations with pain and physical function}

In line with the studies using US, Haugen and colleagues [54] found a significant association between synovitis and tenderness upon palpation, and the association was independent of other MRI features. This study is also the first to demonstrate an association between BMLs and pain in hand OA; together with synovitis, BML is the MRI feature that is most consistently associated with pain in knee OA [43]. Significant associations with pain were also shown for bone damage. Kwok and colleagues [48] confirmed these findings but did not adjust for the co-occurrence of several MRI features.

Structural features such as bone damage and bone formation seemed to be associated with decreased physical functioning, but the results were not consistent and only weak associations were found. The MCP and thumb base joints were not imaged by MRI, and this may have affected these results [54].

\section{Future perspectives}

An extensive scoring system with an accompanying atlas has been proposed [50]. Validation studies have shown that MRI is more sensitive than CR in detection of erosions, suggesting that erosive hand $\mathrm{OA}$ is more common than previously indicated. Future studies should compare marginal erosions on MRI against histology or computer tomography or both, and longitudinal studies should evaluate the predictive value of these lesions. Synovitis and BMLs seem to be associated with pain in hand OA, and the associations should be confirmed in longitudinal studies. The sensitivity to change and the role of MRI as an outcome measure in clinical trials need to be determined. Optimally, further validation will lead to exclusion of less important features from the proposed scoring system, making it more feasible in practice.

\section{Conclusions}

Hand OA is traditionally evaluated by CR. However, CR provides a two-dimensional image of only the bony changes and JSN as an indirect measure of cartilage loss, and the associations between radiographic findings and clinical symptoms are weak to moderate. Indeed, OA is recognized to involve the whole joint, and modern imaging techniques such as US and MRI could be valuable tools for better evaluation of hand OA. US provides a dynamic picture of joint inflammation and can easily be performed during a visit to the rheumatologist. Knowledge of the validity and usefulness of MRI is currently more limited, and the use of MRI in patients with hand OA is currently performed mainly for research purposes.

This article is part of the series Advances in the imaging of rheumatic diseases, edited by Mikkel Østergaard. Other articles in this series can be found at http://arthritis-research.com/series/imaging

\section{Abbreviations}

$\mathrm{BML}$, bone marrow lesion; $\mathrm{CR}$, conventional radiography; DIP, distal interphalangeal; JSN, joint space narrowing; JSW, joint space width; K\&L, Kellgren and Lawrence; MCP, metacarpophalangeal; MRI, magnetic resonance imaging; OA, osteoarthritis; PIP, proximal interphalangeal; US, ultrasonography.

\section{Competing interests}

The authors declare that they have no competing interests.

\section{Authors' contributions}

$\mathrm{IKH}$ reviewed the literature and drafted the manuscript. PB critically revised the manuscript. Both authors read and approved the final manuscript.

\section{Acknowledgments}

We thank Hilde B Hammer for providing the US images.

Published: 13 December 2011

\section{References}

1. Haugen IK, Englund M, Aliabadi P, Niu J, Clancy M, Kvien TK, Felson DT: Prevalence, incidence and progression of hand osteoarthritis in the general population: The Framingham Osteoarthritis Study. Ann Rheum Dis 2011, 70:1581-1586.

2. Maheu E, Altman RD, Bloch DA, Doherty M, Hochberg M, Mannoni A, Punzi L, Spector T, Verbruggen G, Carr A, Cicuttini F, Dreiser RL, Haraoui BP, Hart D, Pelletier J-P, Ramonda R, Rovati L: Design and conduct of clinical trials in patients with osteoarthritis of the hand: recommendations from a task force of the Osteoarthritis Research Society International. Osteoarthritis Cartilage 2006, 14:303-322.

3. Marshall M, Dziedzic KS, van der Windt DA, Hay EM: A systematic search and narrative review of radiographic definitions of hand osteoarthritis in population-based studies. Osteoarthritis Cartilage 2008, 16:219-226.

4. van der Kraan PM, van den Berg WB: Osteophytes: relevance and biology. Osteoarthritis Cartilage 2007, 15:237-244.

5. Kalichman L, Malkin I, Kobyliansky E: Hand bone midshaft enthesophytes: the influence of age, sex, and heritability. Osteoarthritis Cartilage 2007, 15:1113-1119.

6. Gibson N, Guermazi A, Clancy M, Niu J, Grayson P, Aliabadi P, Roemer F, Felson $D$ : Relation of hand enthesophytes with knee enthesopathy: is osteoarthritis related to a systemic enthesopathy? J Rheumato/ 2011, in press.

7. Conaghan PG, Hunter DJ, Maillefert JF, Reichmann WM, Losina E: Summary and recommendations of the OARSI FDA osteoarthritis Assessment of Structural Change Working Group. Osteoarthritis Cartilage 2011, 19:606-610.

8. KwokWY, Kloppenburg M, Rosendaal FR, van Meurs JB, Hofman A, BiermaZeinstra SMA: Erosive hand osteoarthritis: its prevalence and clinical impact in the general population and symptomatic hand osteoarthritis. Ann Rheum Dis 2011, 70:1238-1242.

9. Verbruggen $G$, Veys EM: Numerical scoring systems for the anatomic evolution of osteoarthritis of the finger joints. Arthritis Rheum 1996, 39:308-320.

10. Bijsterbosch J, van Bemmel JM, Watt I, Meulenbelt I, Rosendaal FR, Huizinga TWJ, Kloppenburg M: Systemic and local factors are involved in the evolution of erosions in hand osteoarthritis. Ann Rheum Dis 2011, 70:326-330.

11. Buckland-Wright C: Subchondral bone changes in hand and knee osteoarthritis detected by radiography. Osteoarthritis Cartilage 2004, 12 Suppl A:S10-19.

12. Kellgren JH, Lawrence JS: Radiological assessment of osteo-arthrosis. Ann Rheum Dis 1957, 16:494-502.

13. Kellgren JH, Jeffrey MR, Ball J: The Epidemiology of Chronic Rheumatism. Volume II: Atlas of Standard Radiographs of Arthritis. Oxford: Blackwell Scientific Publications; 1963

14. Lawrence JS: Rheumatism in Populations. London: William Heinemann Medical Books Ltd; 1977.

15. Spector TD, Cooper C: Radiographic assessment of osteoarthritis in population studies: whither Kellgren and Lawrence? Osteoarthritis Cartilage 1993, 1:203-206.

16. Altman R, Hochberg M, Murphy W, Wolfe F, Lequesne M: Atlas of individual radiographic features in osteoarthritis. Osteoarthritis Cartilage 1995, 3:3-70.

17. Altman RD, Gold GE: Atlas of individual radiographic features in 
osteoarthritis, revised. Osteoarthritis Cartilage 2007, 15 Suppl A:A1-56.

18. Maheu E, Cadet C, Gueneugues S, Ravaud P, Dougados M: Reproducibility and sensitivity to change of four scoring methods for the radiological assessment of osteoarthritis of the hand. Ann Rheum Dis 2007, 66:464-469.

19. Bijsterbosch J, Haugen IK, Malines C, Maheu E, Rosendaal FR, Watt I, Berenbaum F, Kvien TK, van der Heijde D, Huizinga TWJ, Kloppenburg M: Reliability, sensitivity to change and feasibility of three radiographic scoring methods for hand osteoarthritis. Ann Rheum Dis 2011, 70:1465-1467.

20. Botha-Scheepers S, Riyazi N, Watt I, Rosendaal FR, Slagboom E, Bellamy N, Breedveld FC, Kloppenburg M: Progression of hand osteoarthritis over 2 years: a clinical and radiological follow-up study. Ann Rheum Dis 2009, 68:1260-1264

21. Bijsterbosch J, Watt I, Meulenbelt I, Rosendaal FR, Huizinga TWJ, Kloppenburg $\mathrm{M}$ : Clinical and radiographic disease course of hand osteoarthritis and determinants of outcome after 6 years. Ann Rheum Dis 2011, 70:68-73.

22. Amin S, LaValley MP, Guermazi A, Grigoryan M, Hunter DJ, Clancy M, Niu J, Gale DR, Felson DT: The relationship between cartilage loss on magnetic resonance imaging and radiographic progression in men and women with knee osteoarthritis. Arthritis Rheum 2005, 52:3152-3159.

23. van't Klooster R, Hendriks EA, Watt I, Kloppenburg M, Reiber JHC, Stoel BC Automatic quantification of osteoarthritis in hand radiographs: validation of a new method to measure joint space width. Osteoarthritis Cartilage 2008, 16:18-25.

24. Huétink K, van 't Klooster R, Kaptein BL, Watt I, Kloppenburg M, Nelissen RGHH, Reiber JHC, Stoel BC: Automatic radiographic quantification of hand osteoarthritis; accuracy and sensitivity to change in joint space width in a phantom and cadaver study. Skeletal Radio/ 2011 Feb 11. [Epub ahead of print].

25. KwokWY, Bijsterbosch J, Malm SH, Biermasz NR, Huetink K, Nelissen RG, Meulenbelt I, Huizinga TWJ, van 't Klooster R, Stoel BC, Kloppenburg M: Validity of joint space width measurements in hand osteoarthritis. Osteoarthritis Cartilage 2011, 19:1349-1355.

26. Dahaghin S, Bierma-Zeinstra SMA, Hazes JMW, Koes BW: Clinical burden of radiographic hand osteoarthritis: a systematic appraisal. Arthritis Rheum 2006, 55:636-647.

27. Kortekaas MC, KwokW-Y, Reijnierse M, Huizinga TWJ, Kloppenburg M: Osteophytes and joint space narrowing are independently associated with pain in finger joints in hand osteoarthritis. Ann Rheum Dis 2011, 70:1835-1837.

28. Summers MN, Haley WE, Reveille JD, Alarcón GS: Radiographic assessment and psychologic variables as predictors of pain and functional impairment in osteoarthritis of the knee or hip. Arthritis Rheum 1988, 31:204-209.

29. Möller I, Bong D, Naredo E, Filippucci E, Carrasco I, Moragues C, lagnocco A: Ultrasound in the study and monitoring of osteoarthritis. Osteoarthritis Cartilage 2008, 16 Suppl 3:S4-7.

30. Keen HI, Wakefield RJ, Grainger AJ, Hensor EMA, Emery P, Conaghan PG: An ultrasonographic study of osteoarthritis of the hand: synovitis and its relationship to structural pathology and symptoms. Arthritis Rheum 2008, 59:1756-1763.

31. Vlychou M, Koutroumpas A, Malizos K, Sakkas LI. Ultrasonographic evidence of inflammation is frequent in hands of patients with erosive osteoarthritis. Osteoarthritis Cartilage 2009, 17:1283-1287.

32. Keen HI, Wakefield RJ, Hensor EMA, Emery P, Conaghan PG: Response of symptoms and synovitis to intra-muscular methylprednisolone in osteoarthritis of the hand: an ultrasonographic study. Rheumatology (Oxford) 2010, 49:1093-1100.

33. Kortekaas MC, KwokW-Y, Reijnierse M, Watt I, Huizinga TWJ, Kloppenburg M: Pain in hand osteoarthritis is associated with inflammation: the value of ultrasound. Ann Rheum Dis 2010, 69:1367-1369.

34. Mancarella L, Magnani M, Addimanda O, Pignotti E, Galletti S, Meliconi R: Ultrasound-detected synovitis with power Doppler signal is associated with severe radiographic damage and reduced cartilage thickness in hand osteoarthritis. Osteoarthritis Cartilage 2010, 18:1263-1268

35. Wittoek R, Carron P, Verbruggen G: Structural and inflammatory sonographic findings in erosive and non-erosive osteoarthritis of the interphalangeal finger joints. Ann Rheum Dis 2010, 69:2173-2176.

36. Keen HI, Lavie F, Wakefield RJ, D'Agostino M-A, Hammer HB, Hensor E, Pendleton A, Kane D, Guerini H, Schueller-Weidekamm C, Kortekaas MC, Birrel F, Kloppenburg M, Stamm T, Watt I, Smolen JS, Maheu E, Dougados M, Conaghan PG: The development of a preliminary ultrasonographic scoring system for features of hand osteoarthritis. Ann Rheum Dis 2008, 67:651-655.
37. Iagnocco A, Filippucci E, Ossandon A, Ciapetti A, Salaffi F, Basili S, Grassi W, Valesini G: High resolution ultrasonography in detection of bone erosions in patients with hand osteoarthritis. J Rheumatol 2005, 32:2381-2383.

38. Wittoek R, Jans L, Lambrecht V, Carron P, Verstraete K, Verbruggen G: Reliability and construct validity of ultrasonography of soft tissue and destructive changes in erosive osteoarthritis of the interphalangeal finger joints: a comparison with MRI. Ann Rheum Dis 2011, 70:278-283.

39. Keen HI, Wakefield RJ, Grainger AJ, Hensor EMA, Emery P, Conaghan PG: Can ultrasonography improve on radiographic assessment in osteoarthritis of the hands? A comparison between radiographic and ultrasonographic detected pathology. Ann Rheum Dis 2008, 67:1116-1120.

40. Möller B, Bonel H, Rotzetter M, Villiger PM, Ziswiler H-R: Measuring finger joint cartilage by ultrasound as a promising alternative to conventional radiograph imaging. Arthritis Rheum 2009, 61:435-441.

41. Iagnocco A, Perella C, D'Agostino MA, Sabatini E, Valesini G, Conaghan PG: Magnetic resonance and ultrasonography real-time fusion imaging of the hand and wrist in osteoarthritis and rheumatoid arthritis. Rheumatology (Oxford) 2011, 50:1409-1413

42. Koutroumpas AC, Alexiou IS, Vlychou M, Sakkas LI: Comparison between clinical and ultrasonographic assessment in patients with erosive osteoarthritis of the hands. Clin Rheumatol 2010, 29:511-516.

43. Yusuf E, Kortekaas MC, Watt I, Huizinga TWJ, Kloppenburg M: Do knee abnormalities visualised on MRI explain knee pain in knee osteoarthritis? A systematic review. Ann Rheum Dis 2011, 70:60-67.

44. Tan AL, Grainger AJ, Tanner SF, Shelley DM, Pease C, Emery P, McGonagle D: High-resolution magnetic resonance imaging for the assessment of hand osteoarthritis. Arthritis Rheum 2005, 52:2355-2365.

45. Peterfy CG, Janzen DL, Tirman PF, van Dijke CF, Pollack M, Genant HK: 'Magicangle' phenomenon: a cause of increased signal in the normal lateral meniscus on short-TE MR images of the knee. AJR Am J Roentgenol 1994, 163:149-154.

46. Haugen IK, Bøyesen P, Slatkowsky-Christensen B, Sesseng S, Bijsterbosch J, van der Heijde D, Kvien TK: Comparison of features by MRI and radiographs of the interphalangeal finger joints in patients with hand osteoarthritis. Ann Rheum Dis 2011 Oct 11. [Epub ahead of print].

47. Baker K, Grainger A, Niu J, Clancy M, Guermazi A, Crema M, Hughes L, Buckwalter J, Wooley A, Nevitt M, Felson DT: Relation of synovitis to knee pain using contrast-enhanced MRIs. Ann Rheum Dis 2010, 69:1779-1783.

48. KwokWY, Kortekaas MC, Reijnierse M, van der Heijde D, Bloem JL, Kloppenburg M: MRI in hand osteoarthritis: Validation of the Oslo hand osteoarthritis MRI-scoring method and association with pain. Osteoarthritis Cartilage 2011, 19 Suppl 1:S26-S27.

49. McQueen F, Østergaard M, Peterfy C, Lassere M, Ejbjerg B, Bird P, O'Connor P, Genant H, Shnier R, Emery P, Edmonds J, Conaghan P: Pitfalls in scoring MR images of rheumatoid arthritis wrist and metacarpophalangeal joints. Ann Rheum Dis 2005, 64 Suppl 1:i48-55.

50. Haugen IK, Lillegraven S, Slatkowsky-Christensen B, Haavardsholm EA, Sesseng S, Kvien TK, Van Der Heijde D, Bøyesen P: Hand osteoarthritis and MRI: development and first validation step of the proposed Oslo Hand Osteoarthritis MRI score. Ann Rheum Dis 2011, 70:1033-1038.

51. Lewis AR, Nolan MJ, Hodgson RJ, Benjamin M, Ralphs JR, Archer CW, Tyler JA, Hall LD: High resolution magnetic resonance imaging of the proximal interphalangeal joints. Correlation with histology and production of a three-dimensional data set. J Hand Surg Br 1996, 21:488-495.

52. Tan AL, Toumi H, Benjamin M, Grainger AJ, Tanner SF, Emery P, McGonagle D: Combined high-resolution magnetic resonance imaging and histological examination to explore the role of ligaments and tendons in the phenotypic expression of early hand osteoarthritis. Ann Rheum Dis 2006, 65:1267-1272.

53. Grainger AJ, Farrant JM, O'Connor PJ, Tan AL, Tanner S, Emery P, McGonagle D: MR imaging of erosions in interphalangeal joint osteoarthritis: is all osteoarthritis erosive? Skeletal Radiol 2007, 36:737-745.

54. Haugen IK, Bøyesen P, Slatkowsky-Christensen B, Sesseng S, van der Heijde D, Kvien TK: Associations between MRI-defined synovitis, bone marrow lesions and structural features and measures of pain and physical function in hand osteoarthritis. Ann Rheum Dis 2011, in press.

doi:10.1186/ar3509

Cite this article as: Haugen IK, Bøyesen P: Imaging modalities in hand osteoarthritis - status and perspectives of conventional radiography, magnetic resonance imaging, and ultrasonography. Arthritis Research \& Therapy 2011, 13:248. 